\section{$1 \mathrm{P341}$}

Single Giant Unilamellar Vesicle Method Reveals Interaction of (-)Epigallocatechin Gallate, Tea Catechin, with Lipid Membranes

Yukihiro Tamba ${ }^{1}$, OShinya Ohba ${ }^{2}$, Hiroe Yoshioka ${ }^{3}$, Masahito Yamazaki ${ }^{4}$

${ }^{1}$ IJRC., Shizuoka Univ., ${ }^{2}$ Dept. Phys., Fac. Sci., Shizuoka Univ., ${ }^{3}$ RRC., Fac.Sci, Shizuoka Univ., ${ }^{4}$ Int. Biosci. Sec., Grad. Sch. of Sci. and Eng., Shizuoka Univ.

Tea catechins have been considered to have antibacterial activity and antioxidant. Several investigations indicated that lipid membranes are one of the targets of catechins for their activities. However, the detail interaction of catechins with lipid membranes remains unclear. In this report, we investigated the interaction of (-)-epigallocatechin gallate (EGCg), a dominant catechin in tea extract, with single giant unilamellar vesicles (GUVs) of egg phosphatidylcholine (egg PC) by phase contrast, fluorescence microscopy using the single GUV method.

Low concentrations of EGCg $(-60 \mu \mathrm{M})$ induced a rapid leakage of a fluorescent probe, calcein, from the inside of single egg PC-GUVs, which changed into small lumps of lipids after the leakage. We found the detailed process of the EGCg-induced burst of GUVs, the decrease in its diameter, and the transformation into the small lumps. These results indicate that the leakage of calcein occurred as a result of the burst of the GUV. The fraction of the burst GUV increased with time, and also with an increase in EGCg concentration. The analysis of the EGCginduced shape changes shows that binding of EGCg to the external monolayer of the GUV increases its membrane area, inducing an increase in its surface pressure. Small-angle $\mathrm{X}$-ray scattering experiments indicated that the intermembrane distance of multilamellar vesicles of DOPC membrane greatly decreased above the threshold EGCg concentrations. On the basis of these results, we discuss the mechanism of the EGCg-induced burst of vesicles.

\section{$1 \mathrm{P343}$}

\section{Several factors affecting the pore formation of Antimicrobial Peptide, Magainin 2}

OYukihiro Tamba ${ }^{1}$, Takuya Yoshitani ${ }^{2}$, Junji Mano ${ }^{2}$, Masahito Yamazaki ${ }^{3}$

${ }^{1}$ IJRC, Shizuoka Univ., ${ }^{2}$ Dept. of Phys., Fac. of Sci., Shizuoka Univ., ${ }^{3}$ Int. Biosci. Sec., Grad. Sch. of Sci. and Eng., Shizuoka Univ.

It is considered that the target of magainin 2, an antimicrobial peptide, is lipid membranes. Recently we investigated the interactions of magainin 2 with dioleoylphosphatidylglycerol and dioleoylphosphatidylcholine (DOPG/DOPC(5/5)) membranes using the single GUV(giant unilamellar vesicle) method [1]. We have found that the leakage of calcein from a GUV occurred through the pore formed by magainin 2 in the membrane and that the fraction of completely leaked GUV, $P_{\mathrm{L}}(t)$, increased with time and also with an increase magainin 2 concentration. We proposed that the two-state transition model for the pore formation. In this study, we investigated effect of several factors on the magainin 2-induced leakage using the single GUV method

First, we investigated effect of surface charge density of DOPG/DOPC-GUVs. As the surface charge density decreased, the threshold concentration of magainin 2 to induce the leakage increased. For example, magainin 2 concentrations at $P_{\mathrm{L}}(5 \mathrm{~min}$.) $=0.5$ were 3.5 and $50 \mu \mathrm{M}$ for $\mathrm{DOPG} / \mathrm{DOPC}(5 / 5)-\mathrm{GUV}$ and DOPG/DOPC(3/7)-GUV, respectively. Next we investigated the temperature dependence of the $7 \mu \mathrm{M}$ magainin 2-induced leakage from DOPG/DOPC(5/5)GUVs. As temperature increased, $P_{\mathrm{L}}(t)$ increased with time more rapidly. Using this result, we determined the activation energy from the binding state of magainin 2 to the membrane interface of the external monolayer to the pore state in the membrane. On the basis of results of several factors, we discuss the mechanism of the pore formation.

\section{$1 \mathrm{P342}$}

Effects of fusogenic peptides on substrate supported planar lipid bilayers.

OTakehiko Inaba, Yoshiro Tatsu, Kenichi Morigaki AIST

Synthetic analogue peptides derived from the $\mathrm{N}$-terminal region of influenza hemagglutinin are known to be membrane fusogenic. For example, the mixture of these two peptides, E5 and K5, causes fusion of lipid vesicles. The fusion events have been mostly studied by monitoring the lipid mixing with the resonance-energy transfer or by directly observing giant liposomes with dark field microscopy. Although those analyses revealed the occurrence of membrane fusion, the dynamic change of lipid membrane topography caused by peptides has not been clearly understood. As a way for investigating membrane fusion in more detail, we are currently studying the effects of E5 and K5 on substrate supported planar lipid bilayers (SPBs). One unique feature of SPBs compared with lipid vesicles is the accessibility to various analytical techniques that can detect interfacial events with an extremely high sensitivity (e.g. fluorescence recovery after photobleaching (FRAP), quartz crystal microbalance with dissipation monitoring (QCM-D)). We applied FRAP to measure lipid membrane fluidity and QCM-D to measure lipid membrane stability and peptide binding. We report data from these analyses to elucidate effects of fusogenic peptides on SPBs.

\section{$1 \mathrm{P344}$}

Membrane perturbation by antimicrobial peptides from amphibian and arachnid species

OMartin P. Boland ${ }^{1}$, Tara L. Pukala ${ }^{2}$, John D. Gehman', Lucia Kuhn-Nentwig ${ }^{3}$, John H. Bowie ${ }^{2}$, Frances Separovic

${ }^{1}$ School of Chemistry, University of Melbourne, VIC 3010, ${ }^{2}$ Department of Chemistry, University of Adelaide, SA 5005, ${ }^{3}$ Zoological Institute, University of Bern, Baltzerstrasse 6, CH-3012, Bern

Increasing resistance to antibiotics has led to investigation of alternative antimicrobial compounds, including cationic host defense peptides isolated from frogs and spiders. Their mode of action is postulated to be related to the structure and length of the peptide. Shorter peptides that adopt a linear $\alpha$-helical structure in membrane mimetic environments are believed to act via membrane solubilizing mechanisms, e.g. aurein 1.2 and citropin 1.1 from Australian tree frogs (13 and 16 residues, respectively). Longer peptides, e.g. maculatin 1.1 and cupiennin 1a (21 and 35 residues, respectively, with the latter from the spider Cupiennius salei) share a kinked helical structure that is theoretically able to span the lipid bilayer. Solidstate NMR was used to study interactions of the peptides with model membrane systems. Zwitterionic phospholipid bilayers of dimyristoylphosphatidylcholine were used to represent the cell membranes of eukaryotes, while membranes containing the anionic phospholipid dimyristoylphosphatidylglycerol were used as a model of a bacterial cell membrane. Addition of the peptides modulated the ${ }^{2} \mathrm{H}$ and ${ }^{31} \mathrm{P}$ NMR spectra of phospholipid bilayers. Changes were observed in the ${ }^{31} \mathrm{P}$ relaxation rates and the segmental order profile of the acyl chains. These peptides have previously been shown to have a low affinity for neutral phospholipids whereas our new results indicate preferential binding to membranes with a net negative charge and suggest a lytic mechanism for bacterial outer membranes.

[1] Biochemistry, 44, 15823, 2005 